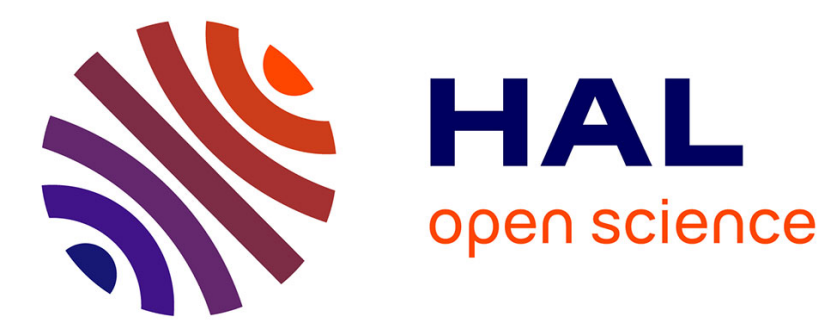

\title{
Numerical schemes for low Mach wave breaking
}

Frederic Golay, Philippe Helluy

\section{To cite this version:}

Frederic Golay, Philippe Helluy. Numerical schemes for low Mach wave breaking. International Journal of Computational Fluid Dynamics, 2007, 21 (2), pp.69-86. 10.1080/10618560701343382 . hal-00139634

\section{HAL Id: hal-00139634 \\ https://hal.science/hal-00139634}

Submitted on 3 Apr 2007

HAL is a multi-disciplinary open access archive for the deposit and dissemination of scientific research documents, whether they are published or not. The documents may come from teaching and research institutions in France or abroad, or from public or private research centers.
L'archive ouverte pluridisciplinaire HAL, est destinée au dépôt et à la diffusion de documents scientifiques de niveau recherche, publiés ou non, émanant des établissements d'enseignement et de recherche français ou étrangers, des laboratoires publics ou privés. 


\title{
NUMERICAL SCHEMES FOR LOW MACH WAVE BREAKING
}

\author{
FRÉDÉRIC GOLAY AND PHILIPPE HELLUY
}

\begin{abstract}
In this work, we describe a finite volume scheme for the computation of incompressible air-water flows. We use an artificial compressibility approach that permits us to use a completely explicit scheme. We describe successively the low Mach preconditioning of the scheme, the Riemann solver and the non-conservative approach that is used to suppress velocity-pressure oscillations, the second order extensions and the parallel implementation. Then it is applied to the simulation of the breaking of a wave on a $15 \%$ slope.
\end{abstract}

\section{INTRODUCTION}

The numerical simulation of wave breaking has a long history (for a survey by S. Grilli, see [17]). There are mainly two methods of approximation:

- The Boundary Integral Elements Method (BIEM) relies on a hypothesis of potential flow. The Poisson equation on the potential under the free surface is transformed into a non-linear, time-dependant integral equation thanks to the Green function. The method is quite efficient and permits a computation up to the reconnection of the jet with high precision [14].

- The other method relies on the resolution of the complete incompressible Navier-Stokes equations coupled with a special treatment at the free surface. It is more general and also valid after the reconnection. If the treatment of the air-water interface is based on moving mesh techniques (Lagrangian approach), it is called a front tracking method. If the computation is performed on a fixed grid, it falls in the category of front capturing methods. Examples of front capturing methods for this king of flows can be found in many papers as, for examples, [28], [15], [5], etc.

In this paper, we study an original front capturing method for computing general incompressible air-water flows and apply it to a $2 \mathrm{D}$ wave breaking over a reef. The main idea is to take into account compressibility effects in the model, even if the flow is clearly incompressible. The main advantage of this approach is that it is possible to avoid implicit schemes in the computation of the pressure, as it is usual in classical incompressible approaches. We have already tested successfully the method in a previous paper [17], including comparisons with experiments and other methods. Artificial compressibility methods have been already widely employed in wave breaking simulation [15], [6], etc. The two novelties are: a physical low Mach number preconditioning and the use of a non-conservative scheme in order to improve the precision.

1991 Mathematics Subject Classification. 76M12, 65M12.

Key words and phrases. finite volume, low Mach number, parallel computing, two-phase flows, wave breaking. 
We concentrate here on several improvements of the method in order to envisage realistic 3D computations. In [17] the mesh was regular, we use here curvilinear meshes. The finite volume Godunov scheme that is implemented would be convenient for arbitrary unstructured meshes but we use here the Cartesian topology of the mesh to avoid the storage of the gradients of the unknowns at the centroids of the control volumes. The Cartesian mesh also permits an easier parallel implementation of the scheme. In [17], we also solved the energy conservation law. We will try to avoid this computation by employing an isothermal model.

The paper is then organized as follows:

- In Section 1, we first state two mathematical models for compressible airwater flows: an isothermal Euler model ("isothermal model") and a complete Euler model with an energy conservation law ("energy model"). We show how it is possible to compute low Mach flows by an adequate tuning of the pressure law coefficients. This physical "preconditioning" is crucial to avoid too constraining CFL conditions and to achieve acceptable precision. We show that the two models lead to a well-posedness of the Riemann problem that is at the heart of the Godunov finite volume method.

- In Section 2 we recall, thanks to 1D experiments, that the classical conservative finite volume schemes have a very bad behavior at the air-water interface (pressure oscillations). We adapt a trick by Abgrall and Saurel (described in [1], [2], [3], [9]) in order to avoid these oscillations in the energy model. The trick is based on a non-conservative resolution of the air fraction evolution. We show that the trick also works with our isothermal model, thanks to the linearity of the pressure law.

- Section 3 is devoted to the presentation of the finite volume scheme. We first present the first order version, together with the non-conservative correction. We also introduce several second order extensions based on the MUSCL ${ }^{1}$ Van Leer approach [27]. Two slope limiters are tested: the Barth limiter described in many textbooks (as [10]) and the WLSQR ${ }^{2}$ limiter. The WLSQR limiter is a variant of the WENO ${ }^{3}$ limiter. It is described in [7] and included references. For the time integration we propose to test two techniques: the MUSCL-Hancock approach [24] and the classical midpoint Euler approach.

- Section 4 is devoted to several 1D and 2D experiments: "shock tube" test cases, the propagation of a stable solitary wave over a flat bottom and finally the wave breaking of a solitary wave arriving on a $15 \%$ slope.

\section{Mathematical MODEL}

1.1. Compressible model for two-fluid flows. We wish to compute a two-fluid air-water flow in a three-dimensional box $\Omega$ defined by

$$
\mathbf{x}=\left(x_{1}, x_{2}, x_{3}\right)=(x, y, z) \in \Omega \Leftrightarrow\left\{\begin{array}{c}
x_{\min }<x<x_{\max }, \\
y_{\min }<y<y_{\max }, \\
z_{\min }+b(x, y)<z<z_{\max } .
\end{array}\right.
$$

\footnotetext{
${ }^{1}$ MUSCL stands for Monotonic Upwind Scheme for Conservation Laws

${ }^{2}$ WLSQR stands for Weighted Least SQUare

${ }^{3}$ WENO stands for Weighted Essentially Non Oscillatory
} 
The unknowns are the density $\rho$, the velocity vector

$$
\mathbf{u}=\left(u_{1}, u_{2}, u_{3}\right)=(u, v, w)
$$

the pressure $p$ and the air fraction $\varphi$ of the two-fluid flow. The unknowns depend on the position $\mathbf{x} \in \Omega$ and the time $t \in[0, T]$.

The air fraction $\varphi$ satisfies

$$
\begin{aligned}
& 0 \leqslant \varphi \leqslant 1, \\
& \varphi(\mathbf{x}, t)=1 \quad \text { if } \mathbf{x} \text { is in the air at time } t \\
& \varphi(\mathbf{x}, t)=0 \quad \text { if } \mathbf{x} \text { is in the water at time } t .
\end{aligned}
$$

We consider two compressible Euler models with gravity. The first model assumes that the flow is isothermal. Considering the conservation laws for the mass and the momentum and the convection of the fraction, it reads

$$
\begin{aligned}
& \rho_{t}+(\rho u)_{x}+(\rho v)_{y}+(\rho w)_{z}=0 \\
& (\rho u)_{t}+\left(\rho u^{2}+p\right)_{x}+(\rho u v)_{y}+(\rho u w)_{z}=0, \\
& (\rho v)_{t}+(\rho v u)_{x}+\left(\rho v^{2}+p\right)_{y}+(\rho v w)_{z}=0, \\
& (\rho w)_{t}+(\rho w u)_{x}+(\rho w v)_{y}+\left(\rho w^{2}+p\right)_{z}=-\rho g, \\
& \varphi_{t}+u \varphi_{x}+v \varphi_{y}+w \varphi_{z}=0, \\
& p=p(\rho, \varphi) .
\end{aligned}
$$

where $g$ denotes the gravity $\left(g=9.81 \mathrm{~m} \cdot \mathrm{s}^{-2}\right)$. The pressure $p=p(\rho, \varphi)$ is a function of the density $\rho$ and the fraction $\varphi$ that will be discussed in Section 1.2.

The second model also considers the energy conservation law. It reads

$$
\begin{aligned}
& \rho_{t}+(\rho u)_{x}+(\rho v)_{y}+(\rho w)_{z}=0, \\
& (\rho u)_{t}+\left(\rho u^{2}+p\right)_{x}+(\rho u v)_{y}+(\rho u w)_{z}=0, \\
& (\rho v)_{t}+(\rho v u)_{x}+\left(\rho v^{2}+p\right)_{y}+(\rho v w)_{z}=0, \\
& (\rho w)_{t}+(\rho w u)_{x}+(\rho w v)_{y}+\left(\rho w^{2}+p\right)_{z}=-\rho g, \\
& (\rho E)_{t}+((\rho E+p) u)_{x}+((\rho E+p) v)_{x}+((\rho E+p) w)_{x}=-\rho g w, \\
& \varphi_{t}+u \varphi_{x}+v \varphi_{y}+w \varphi_{z}=0, \\
& E=\varepsilon+\frac{1}{2}\left(u^{2}+v^{2}+w^{2}\right), \quad p=p(\rho, \varepsilon, \varphi) .
\end{aligned}
$$

The total specific energy $E$ is the sum of the internal specific energy $\varepsilon$ and the specific kinetic energy. The pressure law $p=p(\rho, \varepsilon, \varphi)$ will be discussed in Section 1.2. The two above models can be written in a unified way

$$
\mathbf{W}_{t}+\mathbf{F}(\mathbf{W})_{x}+\mathbf{G}(\mathbf{W})_{y}+\mathbf{H}(\mathbf{W})_{z}=\mathbf{S}(\mathbf{W})
$$


In the first case, the conservative variables vector $\mathbf{W}$, the fluxes $\mathbf{F}, \mathbf{G}, \mathbf{H}$ and the source $\mathbf{S}$ are defined by

$$
\begin{aligned}
& \mathbf{W}=(\rho, \rho u, \rho v, \rho w, \rho \varphi)^{T}, \\
& \mathbf{F}=\left(\rho u, \rho u^{2}+p, \rho v u, \rho w u, \rho \varphi u\right)^{T}, \\
& \mathbf{G}=\left(\rho v, \rho v u, \rho v^{2}+p, \rho v w, \rho \varphi v\right)^{T}, \\
& \mathbf{H}=\left(\rho w, \rho w u, \rho w v, \rho w^{2}+p, \rho \varphi w\right)^{T}, \\
& \mathbf{S}=(0,0,0,-\rho g, 0)^{T}, \\
& p=p(\rho, \varphi) .
\end{aligned}
$$

In the second case, they are defined by

$$
\begin{aligned}
& \mathbf{W}=(\rho, \rho u, \rho v, \rho w, \rho E, \rho \varphi)^{T}, \\
& \mathbf{F}=\left(\rho u, \rho u^{2}+p, \rho v u, \rho w u,(\rho E+p) u, \rho \varphi u\right)^{T}, \\
& \mathbf{G}=\left(\rho v, \rho v u, \rho v^{2}+p, \rho v w,(\rho E+p) v, \rho \varphi v\right)^{T}, \\
& \mathbf{H}=\left(\rho w, \rho w u, \rho w v, \rho w^{2}+p,(\rho E+p) w, \rho \varphi w\right)^{T}, \\
& \mathbf{S}=(0,0,0,-\rho g,-\rho g w, 0)^{T}, \\
& p=p(\rho, \varepsilon, \varphi) .
\end{aligned}
$$

Very few theoretical results are established for the general system of conservation law (5). It is generally admitted (and proved in very particular situations) that existence, uniqueness and stability hold if the system is hyperbolic. We introduce the notation

$$
\begin{aligned}
& \mathbf{n}=\left(n_{1}, n_{2}, n_{3}\right) \\
& \mathbf{F}(\mathbf{V}, \mathbf{n}):=\mathbf{F}(\mathbf{V}) \cdot n_{1}+\mathbf{G}(\mathbf{V}) \cdot n_{2}+\mathbf{H}(\mathbf{V}) \cdot n_{3}
\end{aligned}
$$

The hyperbolicity condition states that for any unit vector $\mathbf{n}=\left(n_{1}, n_{2}, n_{3}\right)$ the matrix

$$
\frac{D \mathbf{F}}{D \mathbf{W}}(\mathbf{W}, \mathbf{n})=\mathbf{A}(\mathbf{W}, \mathbf{n})
$$

is diagonalizable, with real eigenvalues. In the two above models (3) and (4), the computation of the eigenvalues is a very classical exercise (see for example [10]). The eigenvalues are $\mathbf{u} \cdot \mathbf{n}-c, \mathbf{u} \cdot \mathbf{n}$ and $\mathbf{u} \cdot \mathbf{n}+c$ where $c$ is the sound speed of the two-fluid mixture. Even when the pressure law depends on the fraction $\varphi$, the sound speed is defined by the usual formula (where, as before, $p_{\rho}$ and $p_{\varepsilon}$ denote the partial derivatives of $p$ with respect to $\rho$ and $\varepsilon$ )

$$
c^{2}=p_{\rho}+\frac{p}{\rho^{2}} p_{\varepsilon}
$$

For the isothermal model (3), where the pressure law does not depend on $\varepsilon$, it becomes

$$
c^{2}=p_{\rho}(\rho, \varphi)
$$

According to (10), the hyperbolicity condition can also be written

$$
p_{\rho}+\frac{p}{\rho^{2}} p_{\varepsilon}>0
$$

Finally, the Partial Differential Equations (PDE) system has to be supplemented by boundary conditions. In the two cases here, we consider perfect wall conditions 
on the boundary $\partial \Omega$. Considering the outwards unit normal vector $\mathbf{n}=\left(n_{1}, n_{2}, n_{3}\right)$ to $\Omega$ on $\partial \Omega$, this wall condition reads

$$
\mathbf{u} \cdot \mathbf{n}=0 .
$$

1.2. Pressure law. The flows that we wish to simulate are low Mach number flows. The local Mach number $M$ of the flow is the ratio

$$
\begin{aligned}
M & =\frac{|\mathbf{u}|}{c}, \\
|\mathbf{u}| & =\sqrt{u^{2}+v^{2}+w^{2}},
\end{aligned}
$$

where $c$ is the local sound speed defined by (10).

When the Mach number is uniformly small, the flow is almost incompressible. In physics, one usually considers that a flow is incompressible if the Mach $M<0.3$. There will not be big differences between a flow at Mach $M \simeq 1 / 10$ and a flow at Mach $M \simeq 1 / 1000$. In our configurations the material velocity $|\mathbf{u}|$ will be of the order of $1 \mathrm{~m} / \mathrm{s}$. The real sound speed in air is around $400 \mathrm{~m} / \mathrm{s}$ and the real sound speed in water is around $1600 \mathrm{~m} / \mathrm{s}$. If one is interested only in the incompressible flow, it is allowed to set an artificial sound speed in the two fluids. This fact will be used in the sequel for two main reasons:

- avoid too constraining CFL stability condition;

- limit the numerical viscosity and the bad low Mach behavior of the finite volume schemes as described in [16] or [25].

Typically, we will set the sound speed $c$ to the value

$$
c_{0}=20 \mathrm{~m} / \mathrm{s}
$$

for air and water at a reference pressure and density. The reference states are for the water

$$
\rho_{W}=1000 \mathrm{~kg} / \mathrm{m}^{3}, \quad p_{0}=10^{5} \mathrm{~Pa},
$$

and for the air

$$
\rho_{A}=1 \mathrm{~kg} / \mathrm{m}^{3}, \quad p_{0}=10^{5} \mathrm{~Pa} .
$$

Remark 1. Let us note that in our computations the material velocity will typically be of the order of $1 \mathrm{~m} / \mathrm{s}$. Thus it is dangerous to take a smaller sound speed that could exaggerate the compressibility effects. On the other hand a higher sound speed would dramatically increase the numerical diffusion and the CFL constrain.

In the case of the isothermal flow, we choose the pressure law

$$
p=c_{0}^{2}\left(\rho-\left(\varphi \rho_{A}+(1-\varphi) \rho_{W}\right)\right)+p_{0} .
$$

In the case $\varphi=0$ (or $\varphi=1$ ), we recover a classical isothermal pressure law for the water (or the air).

Remark 2. Let us note that our pressure law has no physical meaning in the mixture zone $0<\varphi<1$. If we were able to solve exactly the PDE, $\varphi$ would only take the values 0 or 1 . Because of the numerical diffusion, an artificial mixture will appear in the simulations. In the artificial mixture region, the pressures of the two fluids will not equilibrate. 
Remark 3. Several authors (as in [6]) use a more physical approach in the mixture region. By imposing the pressure equilibrium of the two components, they construct a pressure law with a more complex analytical expression.

In the second case, with the energy equation, the pressure law is a so-called stiffened gas equation

$$
p=(\gamma(\varphi)-1) \rho \varepsilon-\gamma(\varphi) \pi(\varphi) .
$$

The stiffened gas pressure law is a simple generalization of the perfect gas pressure law. Indeed, when $\pi(\varphi)=0$, we recover a perfect gas law. The pressure law coefficients have a particular form, proposed by Abgrall and Saurel in [22]

$$
\begin{aligned}
& \frac{1}{\gamma(\varphi)-1}=\varphi \frac{1}{\gamma_{A}-1}+(1-\varphi) \frac{1}{\gamma_{W}-1}, \\
& \frac{\gamma(\varphi) \pi(\varphi)}{\gamma(\varphi)-1}=\varphi \frac{\gamma_{A} \pi_{A}}{\gamma_{A}-1}+(1-\varphi) \frac{\gamma_{W} \pi_{W}}{\gamma_{W}-1} .
\end{aligned}
$$

The parameters $\gamma_{A}, \gamma_{W}, \pi_{A}$ and $\pi_{W}$ are determined in such a way that the sound speed $c=c_{0}=20 \mathrm{~m} / \mathrm{s}$ for the reference states (15) and (16). Using (10), the sound speed is given here by the formula

$$
c=\sqrt{\frac{\gamma(\varphi)(p+\pi(\varphi))}{\rho}} .
$$

In practice, we choose $\gamma_{W}=\gamma_{A}=1.1$ and the sound speed equation written for $\varphi=0$ and $\varphi=1$ gives the two missing parameters $\pi_{A}$ and $\pi_{W}\left(\pi_{A}=-99636 \mathrm{~Pa}\right.$, $\left.\pi_{W}=263636 \mathrm{~Pa}\right)$.

Remark 4. In order to obtain the uniqueness of the solution, the system (4) or (3) has to be supplemented with an entropy condition (see [19], [10]). Now that we have chosen the pressure laws, it is possible to state precisely the entropy condition, which reads

$$
(S)_{t}+\operatorname{div}(\mathbf{T}) \leqslant 0
$$

in a weak sense. Several choices are possible for the entropy $S$ and the entropy flux T. For the energy model (4), we can take

$$
\begin{aligned}
& S=-\rho \ln \left(\frac{\varepsilon-\pi(\varphi) / \rho}{\rho^{\gamma(\varphi)-1}}\right), \\
& \mathbf{T}=S \mathbf{u} .
\end{aligned}
$$

For the isothermal model, we can take

$$
\begin{aligned}
& S=\frac{1}{2} \rho\left(u^{2}+v^{2}+w^{2}\right)+c_{0}^{2} \rho \ln \rho, \\
& \mathbf{T}=S \mathbf{u}+c_{0}^{2} \rho \mathbf{u} .
\end{aligned}
$$

1.3. Riemann problem. The Riemann problem consists in solving the following one-dimensional problem

$$
\begin{aligned}
& \mathbf{W}_{t}+\mathbf{F}(\mathbf{W})_{x}=0, \\
& \mathbf{W}(x, t)=\left\{\begin{array}{l}
\mathbf{W}_{L} \text { if } x<0, \\
\mathbf{W}_{R} \text { if } x>0
\end{array}\right.
\end{aligned}
$$


This solution is supposed to be self-similar

$$
\mathbf{W}(t, x)=\mathbf{R}\left(\frac{x}{t}, \mathbf{W}_{L}, \mathbf{W}_{R}\right) .
$$

The resolution of the Riemann problem for the compressible Euler equations is described in many references. We can cite [24] or [10] for example. It appears that the computation of the solution in the case of a two-fluid flow is very similar. The details are given for example in [3].

As usual, the solution of the Riemann problem is made up of constant states separated by shock waves, rarefaction waves or a contact discontinuity. It is thus of the form

$$
\mathbf{R}\left(\xi, \mathbf{W}_{L}, \mathbf{W}_{R}\right)=\left\{\begin{array}{lll}
\mathbf{W}_{L} & \text { if } & \xi<\lambda_{1}^{-}, \\
\mathbf{W}_{I} & \text { if } & \lambda_{1}^{+}<\xi<\lambda_{2}, \\
\mathbf{W}_{I I} & \text { if } & \lambda_{2}<\xi<\lambda_{3}^{-}, \\
\mathbf{W}_{R} & \text { if } & \lambda_{3}^{+}<\xi
\end{array}\right.
$$

The unknowns are:

- the two intermediate states $\mathbf{W}_{I}$ and $\mathbf{W}_{I I}$;

- the velocities $\lambda_{1}^{-} \leqslant \lambda_{1}^{+}<\lambda_{2}<\lambda_{3}^{-} \leqslant \lambda_{3}^{+}$.

The situation is depicted on Figure 1.

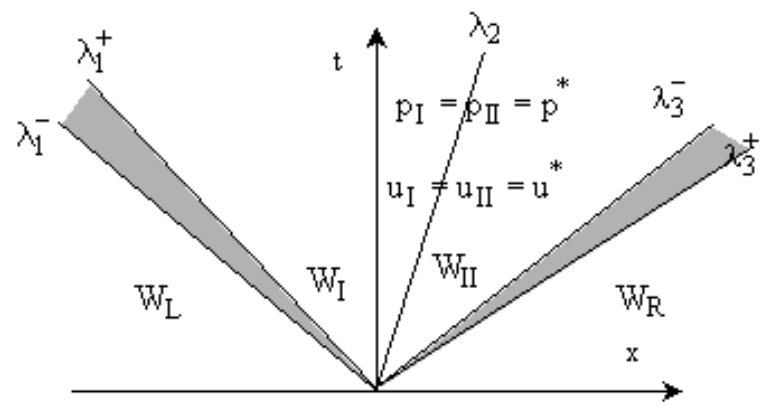

FiguRE 1. Riemann solution in the $(x, t)$ plane.

Furthermore, if $\lambda_{i}^{-}<\lambda_{i}^{+}$(resp. if $\lambda_{i}^{-}=\lambda_{i}^{+}$) then the $i$-wave is a rarefaction wave (resp. a shock of velocity $\sigma=\lambda_{i}^{-}=\lambda_{i}^{+}$). When the $i$-wave is a rarefaction, the computation of $\mathbf{W}=\mathbf{R}\left(\xi, \mathbf{W}_{L}, \mathbf{W}_{R}\right)$, for $\lambda_{i}^{-}<\xi<\lambda_{i}^{+}$is classically carried out by expressing that the three Riemann invariants are constant in the $i$-rarefaction (see $[10])$.

On the other hand, we have $p_{I}=p_{I I}=p^{\star}$. If no vacuum occurs, we can also write $u_{I}=u_{I I}=u^{\star}$. Moreover, it can be verified that the fraction $\varphi$ does not jump in the 1-wave and the 3-wave. It gives $\varphi_{I}=\varphi_{L}$ and $\varphi_{I I}=\varphi_{R}$. It is then classical to compute the 1 - and 3 -waves from the pressure $p^{\star}$ common to the two intermediate states $\mathbf{W}_{I}$ et $\mathbf{W}_{I I}$.

We have now to distinguish between the isothermal model (3) and the energy model (4). 
1.3.1. Isothermal case. In the case of the model (3), we introduce the function

$$
H\left(\rho_{a}, \rho_{b}\right)=\left\{\begin{array}{c}
c_{0} \frac{\rho_{a}-\rho_{b}}{\sqrt{\rho_{a} \rho_{b}}} \text { if } \rho_{a}<\rho_{b}, \\
c_{0} \ln \left(\frac{\rho_{a}}{\rho_{b}}\right) \text { if } \rho_{a}>\rho_{b},
\end{array}\right.
$$

in such a way that the velocities $u_{a}$ and $u_{b}$ on the two sides of a 1-wave (shock or rarefaction) satisfy

$$
u_{b}=u_{a}+H\left(\rho_{a}, \rho_{b}\right) .
$$

In a 3 -wave they have to satisfy a similar relation

$$
u_{b}=u_{a}-H\left(\rho_{a}, \rho_{b}\right) .
$$

The density can be expressed as a linear function of the pressure $p$ and the fraction $\varphi$

$$
\rho(p, \varphi)=\frac{p-p_{0}}{c_{0}^{2}}+\varphi \rho_{A}+(1-\varphi) \rho_{W}
$$

And in the two intermediate states, the pressure is constant equal to $p^{*}$, thus

$$
\begin{aligned}
& \rho_{I}=\rho\left(p^{*}, \varphi_{L}\right), \\
& \rho_{I I}=\rho\left(p^{*}, \varphi_{R}\right) .
\end{aligned}
$$

To solve the Riemann problem, we have first to solve numerically for $p^{*}$

$$
\begin{aligned}
& u^{*}=u_{L}+H\left(\rho_{L}, \rho\left(p^{*}, \varphi_{L}\right)\right) \\
& =u_{R}-H\left(\rho_{R}, \rho\left(p^{*}, \varphi_{R}\right)\right) .
\end{aligned}
$$

It can be proved that equation (29) admits a unique solution

$$
p^{*}>p_{\min }=\max \left(p\left(0, \varphi_{L}\right), p\left(0, \varphi_{R}\right)\right) .
$$

The proof is the same as for a isothermal one-fluid Riemann problem (and can be found in [24], [10]). It relies on the monotony properties of the function

$$
p^{*} \rightarrow u_{R}-u_{L}-H\left(\rho_{R}, \rho\left(p^{*}, \varphi_{R}\right)\right)-H\left(\rho_{L}, \rho\left(p^{*}, \varphi_{L}\right)\right) .
$$

Once $p^{*}$ is known, the rest of the solution can be computed.

1.3.2. Energy model. In the case of the model (4), we introduce the functions

$$
\begin{gathered}
h_{a}\left(p^{\star}\right)=\frac{1}{\rho_{a}} \frac{\left(\gamma_{a}+1\right)\left(p_{a}+\pi_{a}\right)+\left(\gamma_{a}-1\right)\left(p^{\star}+\pi_{a}\right)}{\left(\gamma_{a}+1\right)\left(p^{\star}+\pi_{a}\right)+\left(\gamma_{a}-1\right)\left(p_{a}+\pi_{a}\right)}, \quad a=L \text { or } R, \\
\Phi_{a}\left(p^{\star}\right)=\sqrt{\left(p^{\star}-p_{a}\right)\left(\frac{1}{\rho_{a}}-h_{a}\left(p^{\star}\right)\right),} \\
g_{a}\left(p^{\star}\right)=\frac{1}{\rho_{a}}\left(\frac{p_{a}+\pi_{a}}{p^{\star}+\pi_{a}}\right)^{1 / \gamma_{a}}, \\
\Psi_{a}\left(p^{\star}\right)=\frac{2}{\gamma_{a}-1}\left(\frac{1}{\rho_{a}} \gamma_{a}\left(p_{a}+\pi_{a}\right)\right)^{1 / 2}\left(\left(\frac{p^{\star}+\pi_{a}}{p_{a}+\pi_{a}}\right)^{\left.\frac{\gamma_{a}-1}{2 \gamma_{a}}-1\right),}\right. \\
X_{a}\left(p^{\star}\right)=\left\{\begin{array}{lll}
\Phi_{a}\left(p^{\star}\right) & \text { if } & p^{\star}>p_{a}, \\
\Psi_{a}\left(p^{\star}\right) & \text { if } & p^{\star}<p_{a},
\end{array}\right. \\
H_{a}\left(p^{\star}\right)=\left\{\begin{array}{lll}
h_{a}\left(p^{\star}\right) & \text { if } & p^{\star}>p_{a}, \\
g_{a}\left(p^{\star}\right) & \text { if } & p^{\star}<p_{a} .
\end{array}\right.
\end{gathered}
$$

We then get

$$
u_{I}=u_{L}-X_{L}\left(p^{\star}\right)
$$


and the Riemann problem is solved when $p^{\star}$ is known.

If no vacuum region appears, we have to solve

$$
u^{*}=u_{I}=u_{I I}=u_{L}-X_{L}\left(p^{*}\right)=u_{R}+X_{R}\left(p^{*}\right) .
$$

The following theorem holds

Theorem 1. Let $p_{\min }=-\min \left(\pi\left(\varphi_{L}\right), \pi\left(\varphi_{R}\right)\right)$. If

$$
u_{R}-u_{L} \leq-\left(X_{L}\left(p_{\min }\right)+X_{R}\left(p_{\text {min }}\right)\right),
$$

then the Riemann problem has a unique solution. The pressure $p^{\star} \geq p_{\min }$ is the unique solution of

$$
u_{L}-X_{L}\left(p^{\star}\right)=u_{R}+X_{R}\left(p^{\star}\right) .
$$

This result is quite similar to the case of the Riemann problem for a single fluid. For the proof we refer (for example) to [11], [10], [20]. When inequality (33) is not true, a vacuum has to be introduced. More details and proofs are given in [3]. In practice, for the wave breaking simulations, we did not have to introduce a vacuum to solve the Riemann problem in the finite volume solver for the energy model.

As usual, the equation (29) or (32) on $p^{\star}$ is solved by an iterative Newton method. Typically, we have to numerically solve an equation of the form

$$
f\left(p^{*}\right)=0,
$$

where the function $f$ is strictly monotone, concave and satisfy

$$
\begin{aligned}
& \lim _{p \rightarrow p_{\min }} f(p)=-\infty, \\
& \lim _{p \rightarrow+\infty} f(p)=+\infty .
\end{aligned}
$$

Depending on the initialization, it may happen that a Newton iterate $p$ is smaller than $p_{\min }$. In this case, it is necessary to restart the algorithm with a new $p$ such that

$$
p_{\min }<p \leqslant p^{*} .
$$

\section{LOW PRECISION OF THE CONSERVATIVE APPROACH}

2.1. Failure of the conservative Godunov scheme. This section is devoted to a short and simple presentation of the pressure oscillations phenomenon in the conservative Godunov schemes. It appears that for very simple one-dimensional test cases, the classical first order conservative Godunov scheme gives very bad results on every conservative form of the equations. We first exhibit one of these test cases, which is a simple Riemann problem.

Then, we present a fix proposed by Abgrall and Saurel [1], [2] that permits to avoid the pressure oscillations at the interface. The construction principle is to require that the scheme preserves the moving contact discontinuities. This condition leads to a non-conservative discretization of the transport equation for the air fraction $\varphi$. 
Let us recall that the conservative 1D Godunov scheme also preserves moving contact discontinuities in the case of a one-fluid flow. The non-conservative correction is only useful for multifluid flows. We shall then show that this approach, initially designed for the energy model, can be applied to the isothermal model. Extensions to higher dimensions and higher orders present no difficulty.

In this section, we restrict ourselves to a Riemann problem initial condition. For the numerical experiments, we choose the following values

$$
\mathbf{W}(x, 0)=\left\{\begin{array}{lll}
\mathbf{W}_{L} & \text { if } & x<1 / 2, \\
\mathbf{W}_{R} & \text { if } & x>1 / 2
\end{array}\right.
$$

with

$$
\begin{aligned}
& \gamma_{W}=1.1, \quad \gamma_{A}=1.4, \quad \pi_{W}=\pi_{A}=0 \\
& \rho_{L}=10, \quad u_{L}=50, \quad v_{L}=w_{L}=0, \quad p_{L}=1.1 \times 10^{5}, \quad \varphi_{L}=1 \\
& \rho_{R}=1, \quad u_{R}=50, \quad v_{R}=w_{R}=0, \quad p_{R}=10^{5}, \quad \varphi_{R}=0 .
\end{aligned}
$$

We present numerical results obtained by a classical Godunov scheme. The approximated system is (4) in one dimension written in a conservative form.

Consider a space step $h$ and a time step $\tau$. The discretization points are $x_{i}=$ $i h, \quad i \in Z$. The cells $C_{i}$ are centered on $\left.x_{i}, C_{i}=\right] x_{i-1 / 2}, x_{i+1 / 2}[$. We look for an approximation of $\mathbf{W}$ in the cell $C_{i}$ at time $t_{n}=n \tau$

$$
\mathbf{W}_{i}^{n} \simeq \mathbf{W}\left(t_{n}, x\right), \quad x \in C_{i}
$$

A general conservative finite volumes scheme reads

$$
\mathbf{W}_{i}^{n+1}=\mathbf{W}_{i}^{n}-\frac{\tau}{h}\left(\mathbf{F}_{i+1 / 2}^{n}-\mathbf{F}_{i-1 / 2}^{n}\right)
$$

In the case of the Godunov scheme, the numerical flux is given by the resolution of a Riemann problem at each cell interface $x_{i+1 / 2}$ and takes the form

$$
\mathbf{F}_{i+1 / 2}^{n}=\mathbf{F}\left(\mathbf{R}\left(0, \mathbf{W}_{i}^{n}, \mathbf{W}_{i+1}^{n}\right)\right)
$$

The time step has to satisfy a CFL condition for stability. The CFL number at time $n$ is defined by

$$
\begin{aligned}
& \beta=\frac{\lambda_{\max } \tau}{h}, \\
& \lambda_{\max }=\max _{i} \max \left(\left|u_{i}^{n}-c_{i}^{n}\right|,\left|u_{i}^{n}+c_{i}^{n}\right|\right),
\end{aligned}
$$

where $c_{i}^{n}$ is the sound speed computed by (10) in the cell $i$ at time $n$. We must ensure

$$
\beta<1
$$

The initial conditions are (38). We plot only the pressure at time $t=1 \mathrm{~ms}$. The study interval is ]0, $L$ [ with $L=1 \mathrm{~m}$. The number of cells is fixed at $N=400$ and the CFL number is $\beta=0.7$. We observe pressure oscillations at the contact discontinuity (which is also the material interface between the two fluids). The results are in Figure 2. 


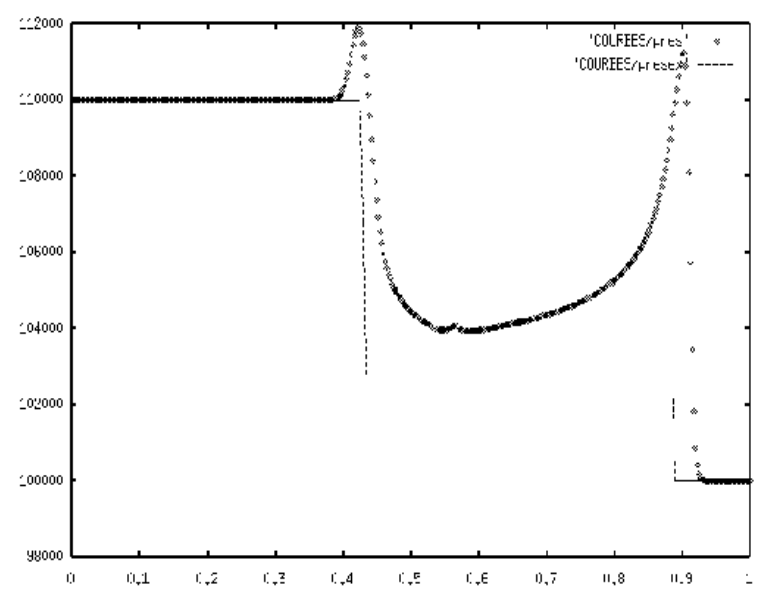

Figure 2. Godunov scheme, pressure (line: exact; dots: numeric). The contact is located at $x=0.55$.

Remark 5. Numerical experiments performed on very fine meshes indicate that the numerical solutions of the classical Godunov scheme indeed converge toward the good solutions when the mesh step $h \rightarrow 0$ (see [9]). The convergence occurs in the $L^{1}$-norm and very seldom in the $L^{\infty}$-norm: overshoots and undershoots are often observed on $\rho, u, p$. Surprisingly, the $\varphi$ variable is often more precise. The numerical rate of convergence is the same as for one-fluid flow computations. An analysis, in the BV space, of these phenomena is given in [4]. Thus it is more rigorous to say that the Godunov conservative scheme suffers from a bad precision instead of "oscillations".

\subsection{A non-conservative Godunov scheme.}

2.2.1. Energy case. The conservative scheme gives very bad results and cannot be used for higher dimensional simulations. This bad behavior is also observed when an approximate Riemann solver is employed. This subject has been studied in many papers: [1], [18], [22], [3], [26], etc. A second order MUSCL extension would slightly improve the results but it is not sufficient.

In order to improve the precision of the Godunov scheme it is possible, as proposed by Saurel and Abgrall in [22], to replace the conservative equation on the fraction

$$
(\rho \varphi)_{t}+(\rho \varphi u)_{x}=0
$$

by its non-conservative equivalent

$$
\varphi_{t}+u \cdot \varphi_{x}=0 .
$$

Although these two equations are equivalent, their approximations may be different. We now show that a special non-conservative approximation give better results. For this, let us consider a general Godunov scheme, associated to an exact or approximate Riemann solver (the VFRoe scheme [8] falls in this category). We suppose that the scheme is conservative for the mass, momentum and energy equations and only give up the conservation of the mass fraction. We index by $i+1 / 2$ the solution of the Riemann problem at the interface beetwen the cell $i$ and the cell $i+1$. 
Suppose that we want to approximate a general moving contact discontinuity of constant velocity $v$ and pressure $p$. To compute the conserved quantities in the cell $i$ at time $n+1$, the scheme reads

$$
\begin{gathered}
\rho_{i}^{n+1}=\rho_{i}^{n}-\frac{\tau}{h}\left((\rho u)_{i+1 / 2}^{n}-(\rho u)_{i-1 / 2}^{n}\right) \\
(\rho u)_{i}^{n+1}=(\rho u)_{i}^{n}-\frac{\tau}{h}\left(\left(\rho u^{2}+p\right)_{i+1 / 2}^{n}-\left(\rho u^{2}+p\right)_{i-1 / 2}^{n}\right) \\
\left(\rho \varepsilon+\rho \frac{u^{2}}{2}\right)_{i}^{n+1}=\left(\rho \varepsilon+\rho \frac{u^{2}}{2}\right)_{i}^{n}-\frac{\tau}{h}\left(\left(\rho \varepsilon u+\rho u \frac{u^{2}}{2}+p u\right)_{i+1 / 2}^{n}\right. \\
\left.-\left(\rho \varepsilon u+\rho u \frac{u^{2}}{2}+p u\right)_{i-1 / 2}^{n}\right) .
\end{gathered}
$$

We now impose that the scheme preserves the moving contact discontinuities, i.e. that $u_{i}^{n+1}=u_{i}^{n}=v$ and $p_{i}^{n+1}=p_{i}^{n}=p$. We obtain

$$
\begin{aligned}
& \rho_{i}^{n+1}=\rho_{i}^{n}-\frac{\tau}{h} v\left(\rho_{i+1 / 2}^{n}-\rho_{i-1 / 2}^{n}\right) \\
& (\rho \varepsilon)_{i}^{n+1}=(\rho \varepsilon)_{i}^{n}-\frac{\tau}{h} v\left((\rho \varepsilon)_{i+1 / 2}^{n}-(\rho \varepsilon)_{i-1 / 2}^{n}\right) .
\end{aligned}
$$

But we have

$$
\rho \varepsilon=p\left(\varphi \frac{1}{\gamma_{A}-1}+(1-\varphi) \frac{1}{\gamma_{W}-1}\right)+\left(\varphi \frac{\gamma_{A} \pi_{A}}{\gamma_{A}-1}+(1-\varphi) \frac{\gamma_{W} \pi_{W}}{\gamma_{W}-1}\right)
$$

We deduce that necessarily, we must have

$$
\varphi_{i}^{n+1}=\varphi_{i}^{n}-\frac{\tau}{h} v\left(\varphi_{i+1 / 2}^{n}-\varphi_{i-1 / 2}^{n}\right) .
$$

This is an upwind approximation of the transport equation (40).

Theorem 2. Any scheme that reduces to (44) for constant velocity and pressure will then preserve moving contact discontinuities.

We can also consider a more general pressure law of the form $p=p_{0}(\rho \varepsilon, \rho, \varphi)$. By inverting this relation, it is possible to express the product $\rho \varepsilon$ as a function of $p, \varphi$ and $\rho$

$$
\rho \varepsilon=g_{0}(p, \varphi, \rho)
$$

Theorem 2 is still true if the function $g_{0}$ is linear with respect to $\varphi$ and $\rho$, for $p$ fixed. This kind of problematic is studied in [9].

We propose now a scheme satisfying Theorem 2. First, we define the interface values by the resolution of Riemann problems at the points $x_{i+1 / 2}$ :

$$
\mathbf{W}_{i+1 / 2}^{n}=R\left(0, \mathbf{W}_{i}^{n}, \mathbf{W}_{i+1}^{n}\right) .
$$

For density, momentum and energy, the classical conservative approach is employed:

$$
\begin{aligned}
\rho_{i}^{n+1} & =\rho_{i}^{n}-\frac{\tau}{h}\left((\rho u)_{i+1 / 2}^{n}-(\rho u)_{i-1 / 2}^{n}\right), \\
(\rho u)_{i}^{n+1} & =(\rho u)_{i}^{n}-\frac{\tau}{h}\left(\left(\rho u^{2}+p\right)_{i+1 / 2}^{n}-\left(\rho u^{2}+p\right)_{i-1 / 2}^{n}\right), \\
(\rho E)_{i}^{n+1} & =(\rho E)_{i}^{n}-\frac{\tau}{h}\left(((\rho E+p) u)_{i+1 / 2}^{n}-((\rho E+p) u)_{i-1 / 2}^{n}\right) .
\end{aligned}
$$

On the other hand, an upwind non-conservative scheme is used for the fraction. This non-conservative scheme is based on the contact discontinuity velocity of the 


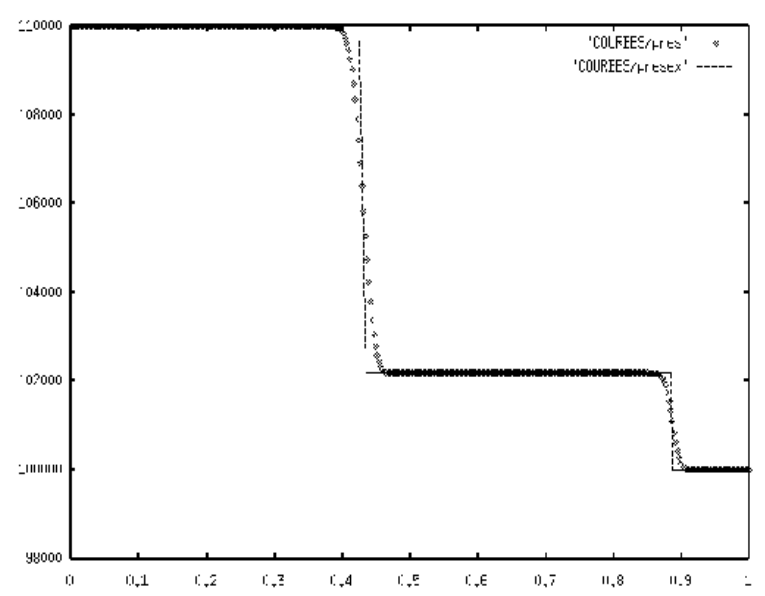

FIGURE 3. Saurel-Abgrall scheme, pressure (line: exact; dots: numeric)

Riemann problems solved at the points $\left(x_{i+1 / 2}\right)$. It reads

$$
\varphi_{i}^{n+1}=\varphi_{i}^{n}-\frac{\tau}{h}\left(\min \left(u_{i+1 / 2}^{n}, 0\right)\left(\varphi_{i+1}^{n}-\varphi_{i}^{n}\right)+\max \left(u_{i-1 / 2}^{n}, 0\right)\left(\varphi_{i}^{n}-\varphi_{i-1}^{n}\right)\right),
$$

It is easy to check that the scheme (46) reduces to (44) for constant velocity and pressure states.

We can also check that our scheme satisfies a maximum principle. Indeed (46) can be rewritten

$$
\begin{aligned}
& \varphi_{i}^{n+1}=a \varphi_{i}^{n}+b \varphi_{i-1}^{n}+c \varphi_{i+1}^{n}, \\
& a=1-b-c \\
& b=\frac{\tau}{h} \max \left(u_{i-1 / 2}^{n}, 0\right), \\
& c=-\frac{\tau}{h} \min \left(u_{i+1 / 2}^{n}, 0\right) .
\end{aligned}
$$

The fraction $\varphi_{i}^{n+1}$ is a convex linear combination of $\varphi_{i}^{n}, \varphi_{i-1}^{n}$ and $\varphi_{i+1}^{n}$ under the CFL condition

$$
\frac{\tau}{h}\left(\max \left(u_{i-1 / 2}^{n}, 0\right)-\min \left(u_{i+1 / 2}^{n}, 0\right)\right) \leqslant 1 .
$$

Our choice is slightly different from the one of Saurel and Abgrall in [22], which is based on the approximate Riemann solver of Rusanov. The Riemann solver of Rusanov is known to be very diffusive. An exact Riemann solver allows us to obtain more precise numerical results, especially in the contact waves.

With the scheme (45), (46), the results on the same test case as above are given in Figure 3. There is an evident improvement.

2.2.2. Isothermal case. Now, we show that this approach can be extended to the isothermal model.

First, we define the interface values by the resolution of Riemann problems at the points $x_{i+1 / 2}$ :

$$
\mathbf{W}_{i+1 / 2}^{n}=\mathbf{R}\left(0, \mathbf{W}_{i}^{n}, \mathbf{W}_{i+1}^{n}\right) \text {. }
$$


For density and momentum, the classical conservative approach is employed:

$$
\begin{aligned}
\rho_{i}^{n+1} & =\rho_{i}^{n}-\frac{\tau}{h}\left((\rho u)_{i+1 / 2}^{n}-(\rho u)_{i-1 / 2}^{n}\right), \\
(\rho u)_{i}^{n+1} & =(\rho u)_{i}^{n}-\frac{\tau}{h}\left(\left(\rho u^{2}+p\right)_{i+1 / 2}^{n}-\left(\rho u^{2}+p\right)_{i-1 / 2}^{n}\right) .
\end{aligned}
$$

On the other hand, an upwind non-conservative scheme is used for the fraction. This non-conservative scheme is based on the contact discontinuity velocity of the Riemann problems solved at the points $\left(x_{i+1 / 2}\right)$. It reads

$$
\varphi_{i}^{n+1}=\varphi_{i}^{n}-\frac{\tau}{h}\left(\min \left(u_{i+1 / 2}^{n}, 0\right)\left(\varphi_{i+1}^{n}-\varphi_{i}^{n}\right)+\max \left(u_{i-1 / 2}^{n}, 0\right)\left(\varphi_{i}^{n}-\varphi_{i-1}^{n}\right)\right)
$$

The scheme preserves constant pressure and velocity states because the density is linear with respect to $\varphi$ when the pressure is fixed.

\section{Finite volume in Higher Dimensions}

3.1. First order scheme. In order to compute the solutions to (4) or (3) we have to detail the finite volume method in higher dimensions. First, the computational domain $\Omega$ is split in several open sets $C_{i}, i \in I=\{1, \cdots, N\}$ called cells or finite volumes such that

(1) $\bigcup_{i \in I} \overline{C_{i}}=\bar{\Omega}$

(2) $\forall(i, j) \in I \times I, i \neq j \Rightarrow C_{i} \cap C_{j}=\emptyset$.

The time domain is also split in a sequence of intervals $] t_{n}, t_{n+1}\left[\right.$ such that $t_{0}=0$, $t_{P}=T, t_{n}<t_{n+1}$. The time step is denoted by $\tau_{n}=t_{n+1}-t_{n}$. The solutions $\mathbf{W}$ are approximated in each cell $C_{i}$ and for each time $t_{n}$ by a constant vector

$$
\mathbf{W}_{i}^{n} \simeq \mathbf{W}\left(\mathbf{x}, t_{n}\right), \quad \mathbf{x} \in C_{i} .
$$

The unknowns satisfy

$$
\int_{C_{i}} \mathbf{W}_{i}^{n+1}=\int_{C_{i}} \mathbf{W}_{i}^{n}-\tau_{n} \int_{\partial C_{i}} \mathbf{F}\left(\mathbf{W}_{i}^{n}, \mathbf{W}_{j}^{n}, \mathbf{n}_{i j}\right)+\tau_{n} \int_{C_{i}} \mathbf{S}\left(\mathbf{W}_{i}^{n}\right) .
$$

In the right hand side of (52), $\mathbf{F}(\mathbf{U}, \mathbf{V}, \mathbf{n})$ is the numerical flux. Index $j$ is for the cells $C_{j}$ that are neighbors of the cell $C_{i}$ along the boundary $\partial C_{i}$ (Figure 4 ). It means that along $\partial C_{i}$ the index $j$ is a piecewise constant function. If the boundary $\partial C_{i}$ has an intersection with $\partial \Omega$, the mirror boundary condition (12) is used in order to extrapolate the outside state $\mathbf{W}_{j}^{n}$. 


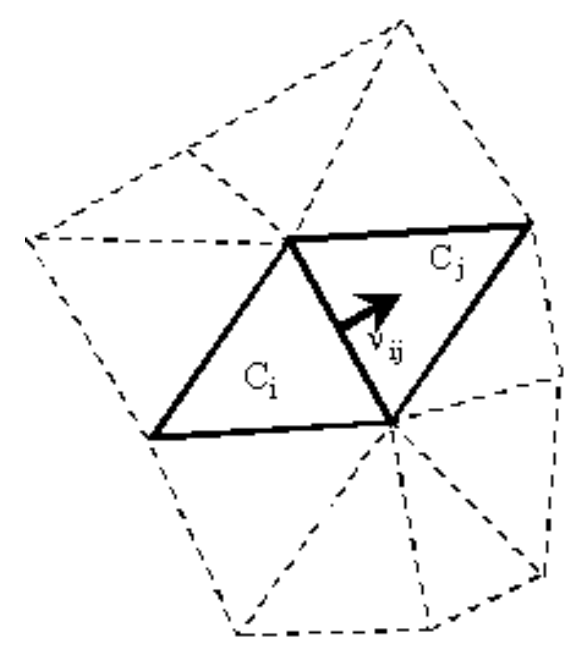

Figure 4. Finite Volume mesh.

An initial condition $\mathbf{W}(\mathbf{x}, 0)=\mathbf{W}_{0}(\mathbf{x})$ is used to start the computation

$$
\int_{C_{i}} \mathbf{W}_{i}^{0}=\int_{C_{i}} \mathbf{W}_{0} .
$$

The vector $\mathbf{n}_{i j}$ is the normal unit vector on $\partial C_{i}$ that points from $C_{i}$ towards $C_{j}$.

The flux $\mathbf{F}\left(\mathbf{W}_{L}, \mathbf{W}_{R}, \mathbf{n}\right)$ is based on an exact resolution of the Riemann problem by neglecting the variations of the solution in the directions orthogonal to $\mathbf{n}$. Using notation (8), the Riemann problem in the direction $\mathbf{n}$ consists in finding a vector valued function $(\xi, t) \rightarrow \mathbf{V}(\xi, t)$ solution of

$$
\begin{aligned}
& \mathbf{V}_{t}+\frac{\partial}{\partial \xi} \mathbf{F}(\mathbf{V}, \mathbf{n})=0, \quad \xi \in R, \quad t>0, \\
& \mathbf{V}(\xi, 0)= \begin{cases}\mathbf{W}_{L} & \text { if } \quad \xi<0, \\
\mathbf{W}_{R} & \text { if } \quad \xi>0 .\end{cases}
\end{aligned}
$$

Thanks to the rotational invariance of the Euler equations, it is equivalent, up to a change of referential, to solve the problem (24). Let us note the unique entropy solution

$$
\mathbf{R}\left(\xi / t, \mathbf{W}_{L}, \mathbf{W}_{R}\right)=\mathbf{V}(\xi, t),
$$

and the solution of the Riemann problem at the interface between $(L)$ and $(R)$ is noted

$$
\mathbf{W}^{*}=\mathbf{W}\left(\mathbf{W}_{L}, \mathbf{W}_{R}\right)=\mathbf{R}\left(0, \mathbf{W}_{L}, \mathbf{W}_{R}\right) .
$$

The classical Godunov flux would be

$$
\mathbf{F}\left(\mathbf{W}_{L}, \mathbf{W}_{R}, \mathbf{n}\right)=\mathbf{F}\left(\mathbf{W}^{*}, \mathbf{n}\right) .
$$

But here, we have to take into account the non-conservative correction. This is done in the following way. We introduce a new vector $\widetilde{\mathbf{W}}$ that is made of the usual conservative variables, except for the last component $\rho \varphi$, which is replaced by the 
non-conservative variable $\varphi$

$$
\begin{aligned}
\widetilde{\mathbf{W}} & =(\rho, \rho u, \rho v, \rho w, \rho E, \varphi)^{T} \quad \text { (energy model) }, \\
\widetilde{\mathbf{W}} & =(\rho, \rho u, \rho v, \rho w, \varphi)^{T} \quad \text { (isothermal model). }
\end{aligned}
$$

The components of the numerical flux $\widetilde{\mathbf{F}}$ are now equal to those of the conservative Godunov flux $\mathbf{F}$, except for the last component, which is now

$$
\widetilde{f}\left(\widetilde{W_{L}}, \widetilde{W_{R}}, \nu\right)_{5} \text { or } 6=\min \left(\mathbf{u}^{*} \cdot \nu, 0\right)\left(\varphi_{R}-\varphi_{L}\right) .
$$

And the scheme (52) is replaced by

$$
\int_{C_{i}} \widetilde{\mathbf{W}}_{i}^{n+1}=\int_{C_{i}} \widetilde{\mathbf{W}}_{i}^{n}-\tau_{n} \int_{\partial C_{i}} \widetilde{\mathbf{F}}\left(\widetilde{\mathbf{W}}_{i}^{n}, \widetilde{\mathbf{W}}_{j}^{n}, \mathbf{n}_{i j}\right)+\tau_{n} \int_{C_{i}} \mathbf{S}\left(\mathbf{W}_{i}^{n}\right) .
$$

Remark 6. For the time integration of the source terms, we have used a very simple scheme. It is known that more sophisticated approaches are required when one needs to capture precisely the rest states. We refer for example to the pioneering work by Leroux [13]. Here we are interested in unsteady computations and the rough classical method is sufficient.

3.2. Second order extensions. It is well known that the first order version of the Godunov scheme has a low precision. It is thus necessary to improve the precision. We shall here use the slope reconstruction technique together with a limitation procedure in order to improve the space accuracy. For the time accuracy, we shall test two methods:

- a second order Runge-Kutta scheme (midpoint Euler);

- the so-called MUSCL Hancock time integration.

Remark 7. In the formula (60), it is clear that the integration of the source term is already second order accurate in space (because the midpoint integration rule is second order accurate).

It is not possible to choose any variable for the slope reconstruction in order to preserve the constant velocity-pressure states. It appears that the reconstruction has good properties in the

$$
\mathbf{Y}=(\rho, u, p, \varphi)
$$

variables for the energy model and in the variables

$$
\mathbf{Y}=(\rho, u, p)
$$

for the isothermal model. We suppose that we have obtained second order approximation of the mean values $\mathbf{W}_{i}^{n}$ of the approximation at time $n$ in the cells $i$. This also gives a second order approximation of the primitive variables $\mathbf{Y}_{i}^{n}=\mathbf{Y}\left(\mathbf{W}_{i}^{n}\right)$ in the centroids of the cells. By a classical slope reconstruction technique, space slopes $\mathbf{s}_{i}^{n}$ and time derivatives $\mathbf{r}_{i}^{n}$ are computed and the primitive variables $\mathbf{Y}$ are now approximated by

$$
\mathbf{Y}^{n+1 / 2}(\mathbf{x})=\mathbf{Y}_{i}^{n}+\mathbf{s}_{i}^{n}\left(\mathbf{x}-\mathbf{x}_{i}\right)+\mathbf{r}_{i}^{n} \frac{\tau_{n}}{2}, \quad \mathbf{x} \in C_{i}
$$




$$
\begin{aligned}
\mathbf{s} & =\left(\begin{array}{lll}
\rho_{x} & \rho_{y} & \rho_{z} \\
\mathbf{u}_{x} & \mathbf{u}_{y} & \mathbf{u}_{z} \\
p_{x} & p_{y} & p_{y} \\
\varphi_{x} & \varphi_{y} & \varphi_{z}
\end{array}\right) \text { (energy model) } \\
\mathbf{s} & =\left(\begin{array}{lll}
\rho_{x} & \rho_{y} & \rho_{z} \\
\mathbf{u}_{x} & \mathbf{u}_{y} & \mathbf{u}_{z} \\
p_{x} & p_{y} & p_{y}
\end{array}\right) \text { (isothermal model) }
\end{aligned}
$$

We can compute the extrapolated values on the cell edges

$$
\mathbf{Y}_{i j}^{n+1 / 2}=\mathbf{Y}_{i}^{n}+\mathbf{s}_{i}^{n}\left(\mathbf{x}_{i j}-\mathbf{x}_{i}\right)+\mathbf{r}_{i}^{n} \frac{\tau_{n}}{2},
$$

where $\mathbf{x}_{i j}$ is the center of the edge between $C_{i}$ and $C_{j}$. Note that, in general,

$$
\mathbf{Y}_{i j} \neq \mathbf{Y}_{j i}
$$

We can also compute the values in the cells at time $n+1 / 2$

$$
\mathbf{Y}_{i}^{n+1 / 2}=\mathbf{Y}_{i}^{n}+\mathbf{r}_{i}^{n} \frac{\tau_{n}}{2}
$$

It is then possible to go back to the variables $\mathbf{W}$ or $\widetilde{\mathbf{W}}$ and compute by an inverse change of variables

$$
\mathbf{W}_{i j}^{n+1 / 2}, \widetilde{\mathbf{W}}_{i j}^{n+1 / 2}, \mathbf{W}_{i}^{n+1 / 2} \text { and } \widetilde{\mathbf{W}}_{i j}^{n+1 / 2} .
$$

Then, we define the interface values by the resolution of Riemann problems at the points $\mathbf{x}_{i j}$ in the direction $\mathbf{n}_{i j}$

$$
\mathbf{W}^{*}=\mathbf{R}\left(0, \mathbf{W}_{i j}^{n+1 / 2}, \mathbf{W}_{j i}^{n+1 / 2}\right) .
$$

The second order scheme now reads

$$
\begin{aligned}
& \int_{\mathbf{C}_{i}} \widetilde{\mathbf{W}}_{i}^{n+1}=\int_{C_{i}} \widetilde{\mathbf{W}}_{i}^{n}-\tau_{n} \int_{\partial C_{i}} \widetilde{\mathbf{F}}\left(\widetilde{\mathbf{W}}_{i j}^{n+1 / 2}, \widetilde{\mathbf{W}}_{j i}^{n+1 / 2}, \mathbf{n}_{i j}\right) \\
& -\tau_{n} \int_{C_{i}}\left(0 \cdots 0, \mathbf{u}_{i}^{n} \cdot \nabla \varphi_{i}^{n}\right)^{T} \\
& +\tau_{n} \int_{C_{i}} \mathbf{S}\left(\mathbf{W}_{i}^{n+1 / 2}\right) .
\end{aligned}
$$

Remark 8. The corrective term

$$
-\tau_{n} \int_{C_{i}}\left(0 \cdots 0, \mathbf{u}_{i}^{n} \cdot \nabla \varphi_{i}^{n}\right)^{T}
$$

is required to achieve second order because the last equation is now non-conservative. Indeed, in contrast with the conservative case, we cannot apply the Stokes formula to transform the volume integral into a surface integral. For the first order scheme, the term (70) cancels because the gradient of the approximate $\varphi$ cancels in each cell. It is no more the case when $\varphi$ is linear in each cell. The second order extension of non-conservative finite volume scheme is studied for example in [21].

In the energy model, the approximated gradient $\nabla \varphi_{i}^{n}$ of $\varphi$ in the cell $C_{i}$ is simply the last line of the tensor $\mathbf{s}_{i}^{n}$ (see (64)).

In the isothermal model, the approximated gradient $\nabla \varphi_{i}^{n}$ is computed from the differentiation of the pressure law (17)

$$
\nabla \varphi=\frac{\nabla p-c_{0}^{2} \nabla \rho}{c_{0}^{2}\left(\rho_{W}-\rho_{A}\right)} .
$$


As before, $\nabla p$ and $\nabla \rho$ are extracted from $\mathbf{s}_{i}^{n}$ in $(64)$.

Using the special forms of the pressure laws (18) and (17), it is easy to check that the second order extension of the scheme still preserves the constant velocitypressure states. Let us remark that it would not be true with other choices of the reconstructed variables than (61) or (62).

Classically, in the MUSCL Hancock reconstruction technique, the space slope is first constructed and limited (two limiters will be given below). It is then easy to give a time slope from the evolution equations on $\mathbf{Y}$. Indeed, $\mathbf{Y}$ is solution of a first order system of the form

$$
\mathbf{Y}_{t}+\sum_{d=1}^{3} \mathbf{B}_{d}(\mathbf{Y}) \mathbf{Y}_{x_{d}}=0
$$

We thus set

$$
\mathbf{r}_{i}^{n}=-\sum_{d=1}^{3} \mathbf{B}_{d}\left(\mathbf{Y}_{i}\right) \mathbf{Y}_{x_{d}}
$$

where the partial derivatives $\mathbf{Y}_{x_{d}}$ are obtained from $\mathbf{s}_{i}^{n}$ in (64).

It is also possible to consider a time first order scheme by setting

$$
\mathbf{r}_{i}^{n}=\mathbf{0}
$$

In this case, the MUSCL scheme present instabilities that develop slowly. In the sequel, if only space slopes are computed, we shall use a second order midpoint Euler integration in time in order to improve the stability. It is easy to check that the midpoint Euler integration does not alter the preservation of constant velocitypressure states.

We now address the several envisaged possibilities for the space slope reconstruction. The first method is due to Barth.

Consider a primitive variable $q$ (any component of $\mathbf{Y}$ ) that has to be reconstructed from the cell values $q_{i}$. First, the gradient is estimated thanks to a discrete version of the Green formula

$$
\operatorname{vol}\left(C_{i}\right) \widetilde{\nabla} q_{i}=\int_{\partial C_{i}} \frac{q_{i}+q_{j}}{2} \mathbf{n}_{i j} .
$$

This gradient has to be limited in order to avoid oscillations. The limiter $\alpha$ is the largest number that satisfies

$$
\begin{aligned}
& 0 \leqslant \alpha \leqslant 1 \\
& \forall j, \quad \alpha\left|\widetilde{\nabla} q_{i} \cdot\left(\mathbf{x}_{i j}-\mathbf{x}_{i}\right)\right| \leqslant\left|q_{j}-q_{i}\right| .
\end{aligned}
$$

The limited gradient is then

$$
\nabla q_{i}=\alpha \tilde{\nabla} q_{i}
$$

The second method is more recent and less diffusive. This is the WLSQR reconstruction technique. The reconstruction in the cell $i$ is a priori sought under the form

$$
q_{i}(x)=q_{i}+\nabla q_{i} \cdot\left(\mathbf{x}-\mathbf{x}_{i}\right) .
$$

Ideally, on the neighbors cell we would like to have

$$
\forall j, \quad \operatorname{vol}\left(C_{j}\right) q_{j}=\int_{C_{j}} q_{i}(\mathbf{x}) .
$$


The system (79) is an overdetermined system of linear equations. We thus decide to solve it in the least square sense. But then, it is known that the resulting scheme will be unstable. Indeed, the whole scheme would be linear and thus cannot be TVD (Total Variation Diminishing) as proven by Goodman and LeVeque in [12].

Instead, we introduce weights noted $\omega_{i j}$ and solve the problem

$$
\nabla q_{i}=\underset{\mathbf{S}}{\arg \min } \sum_{j} \omega_{i j}\left(\int_{C_{j}} q_{i}-q_{j}+\mathbf{S} \cdot\left(\mathbf{x}-\mathbf{x}_{i}\right) d \mathbf{x}\right)^{2} .
$$

The weights are chosen in order to diminish the influence of the $q_{j}$ for which the jump $\left|q_{i}-q_{j}\right|$ is big (shock detection). As suggested by Fürst [7], we choose

$$
\omega_{i j}=\frac{1}{\sqrt{\left|q_{i}-q_{j}\right|+\eta q_{0}}},
$$

where $\eta>0$ is a small parameter and $q_{0}$ is an order of magnitude of the primitive variable $q$.

\section{Application to wave breaking}

4.1. Programming. For the implementation, we have chosen a regular mesh made of hexahedra.

In order to avoid too long computations, we have also implemented a parallel version of the finite volume scheme, using the library MPI (Message Passing Interface). The SGI parallel computer is made of $12 \mathrm{CPUs}$ Itanium II at $1.5 \mathrm{GHz}$. The operating system is Linux RedHat.

The domain is split into $N$ equivalent sub-domains, along the $x$-axis. For the 1D experiments we take $N=1$ and for the 2D validations we take always $N=8$ to speed up the computations.

Because we have in mind an extension to 3D computations, we decided to avoid the storage of the gradients at the centers of the cells. It implies that it is necessary, at the beginning of each time step, to exchange two layers of cells on the left and right faces of the sub-domains.

The implementation is completely $3 \mathrm{D}$ but in this paper we present only $2 \mathrm{D}$ results (only one layer of cells in the $y$ direction).

4.2. 1D validations. In the previous sections, we have proposed two mathematical models for low Mach two-fluid flows. We decided to simulate them by a Godunov scheme. We have now to compare different options in order to select the better compromise in term of CPU time and precision:

- energy or isothermal model;

- Barth or WLSQR limiter;

- midpoint Euler or Hancock time integration.

The comparison is made on 1D academic Riemann problems (shock tubes problem), in all these simple tests, the $y$ and $z$ components of the velocity vector $v=w=0$.

First, we verify that all the schemes preserve the constant velocity-pressure states, which is true for all the options.

The second test consists in a two-fluid Riemann problem. The initial condition is made of two constant states defined as follows:

$$
\begin{aligned}
& u_{L}=0, \quad p_{L}=1.510^{5}, \quad \varphi_{L}=0 \text { if } x<0 \\
& u_{R}=0, \quad p_{R}=1.010^{5}, \quad \varphi_{R}=1 \text { if } x>0 .
\end{aligned}
$$


The density is computed by (28) in the isothermal case and we take $\rho_{L}=1125, \rho_{R}=$ 1 for the energy case. The left and right states are discontinuous at $x=0$. The computational domain is $(x \in[-10,10], y \in[-0.1,0.1], z \in[-0.1,0.1])$, and as already mentioned, we consider a 3D finite volume grid: 100 cells in the $x$-direction and 1 cell in the $y$ or $z$-direction. A mirror condition is imposed on lateral sides. The CFL number is fixed to 0.9 .

The WLSQR scheme is known to be very accurate and robust in the case of single fluid problems [7], though it is CPU time expensive. But in the case of two-fluid problems we have observed that it is often necessary to perform an additional slope limitation at the interface between the two fluids. For these reasons we decided to use only the Barth limiter, which is much faster.

In this computation, we notice a perfect agreement between the energy and the isothermal computations. Thus, in Figure 5 we plot the density along the $x$-axis only for the isothermal model. Except naturally for the first order scheme, all the other results are very similar. In Figure 6, we observe that the left rarefaction wave is best resolved with a first order in time and second order in space approximation.

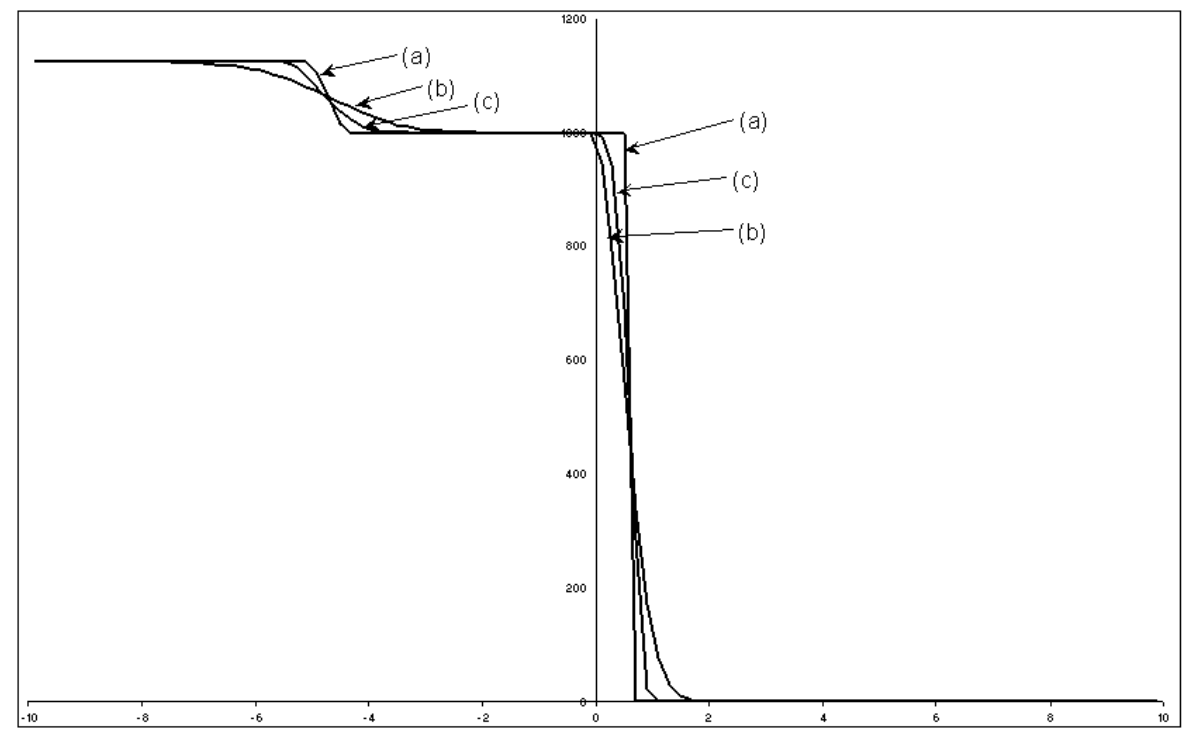

FiguRE 5. Density of the isothermal model at $t=0.25 s:(a)$ exact, (b) first order scheme, (c) Euler Barth scheme 


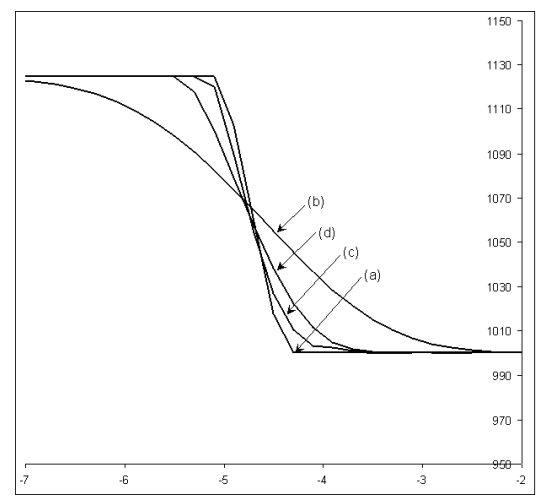

Figure 6. Density of the isothermal model at $t=0.25 \mathrm{~s}$ :(a) exact, (b) first order scheme, (c) Barth scheme, (d) Euler Barth scheme

The accuracy is similar for the other variables $(p$ and $\varphi)$, except for the velocity as shown in Figure 7. We notice many oscillations, especially in the case of the first order in time and second order in space scheme, as shown in Figure 8. This behavior is classical because the MUSCL first order in time and second order in space scheme is linearly unstable.

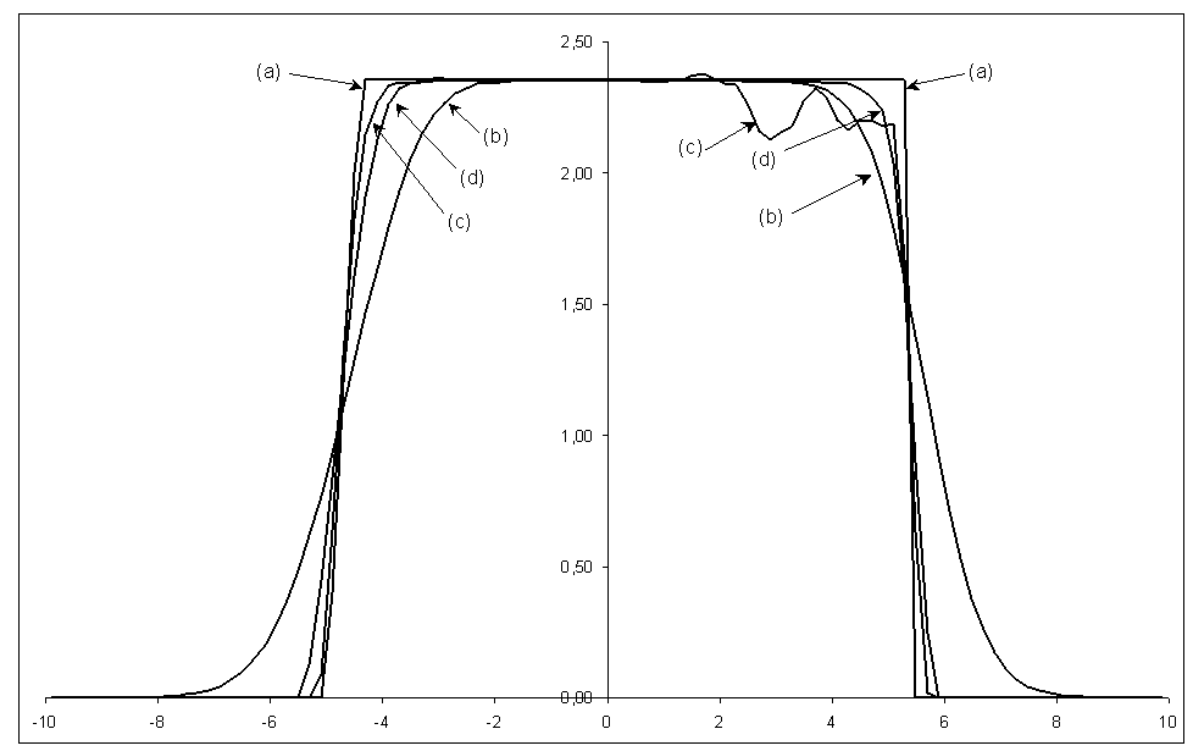

FigURE 7. Velocity of the isothermal model at $t=0.25 s:(a)$ exact, (b) first order scheme, (c) Barth scheme, (d) Euler Barth scheme 


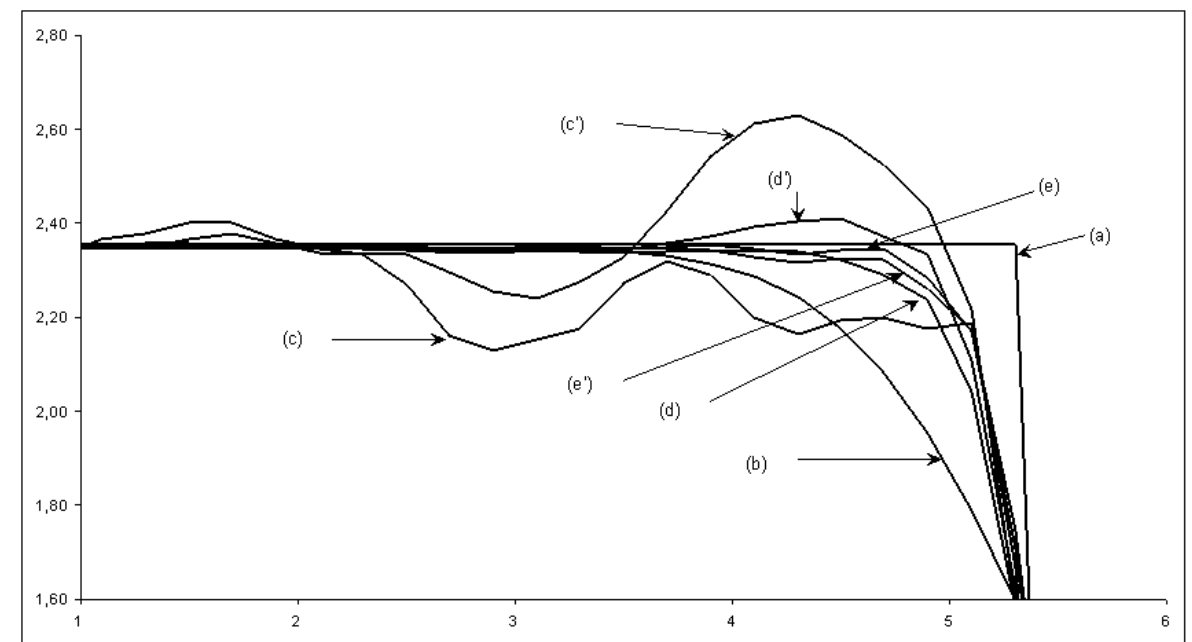

FiguRE 8. Velocity at $t=0.25 s:(a)$ exact, (b) Isothermal first order, (c) Isothermal Barth, (c') Energy Barth, (d) Isothermal Euler Barth , (d') Energy Euler Barth, (e) Isothermal Hancock Barth, (e') Energy Hancock Barth

We observe in Figure 9 that the oscillations vanish with an appropriate mesh refinement.

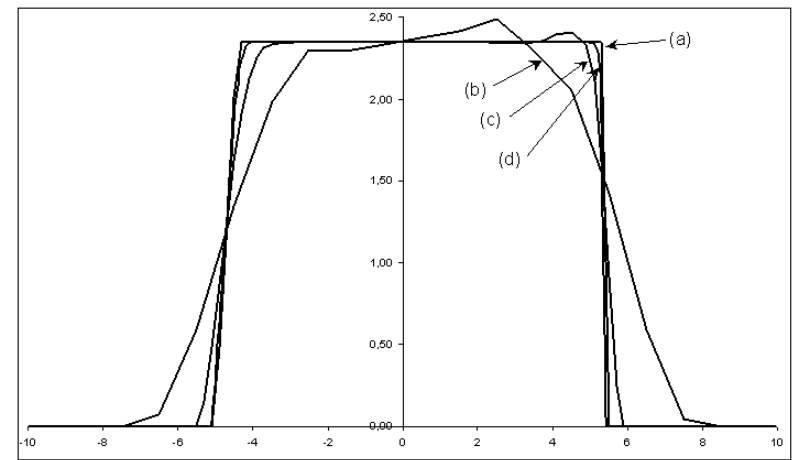

Figure 9. Isothermal, Barth. Velocity at $t=0.25 s:$ (a) exact, (b) 20 cells, (c) 100 cells, (d) 500 cells

The CPU times are compared on Table 1. As expected, the isothermal model corresponds to the shortest computations.

\begin{tabular}{|c|c|c|}
\hline & Isothermal model & Energy model \\
\hline first order & $0.3 \mathrm{~s}$ & $1.1 \mathrm{~s}$ \\
Barth & $0.5 \mathrm{~s}$ & $1.3 \mathrm{~s}$ \\
Euler and Barth & $1.0 \mathrm{~s}$ & $2.5 \mathrm{~s}$ \\
Hancock and Barth & $0.5 \mathrm{~s}$ & $1.3 \mathrm{~s}$ \\
\hline
\end{tabular}

TABLE 1. Cpu time 
4.3. Solitary wave propagation. In this test case we propagate a solitary wave on a flat bottom. The precise boundary and initial conditions are depicted in Figure 10. The initial condition is a stable solitary wave computed thanks to the method of Tanaka [23]: this is an incompressible potential solution of the Euler equations.

The crest of the solitary wave is at $H_{1}=0.6 \mathrm{~m}$ over the still water level. It propagates at a phase velocity $w=1.18 \sqrt{g h_{1}}=3.92 \mathrm{~m} / \mathrm{s}$. The domain $(x \in$ $[-5 ., 22],. y \in[-0.1,0.1], z \in[-1 ., 2]$.$) is approximated by a coarse structured 3D$ finite volume grid: 600 cells in the $x$-direction, 1 cell in the $y$-direction and 90 cells in the $z$-direction. A mirror condition is imposed on the lateral sides. The CFL number is fixed to 0.9 . We propagate this solitary wave during 4 seconds (15.68 $\mathrm{m})$.

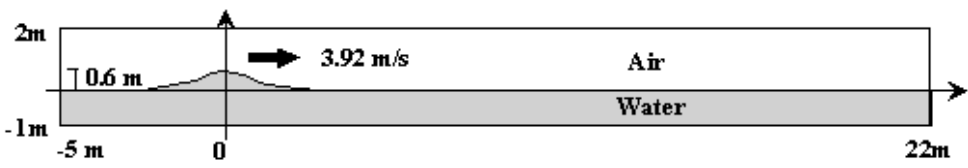

Figure 10. Propagation of a solitary wave with flat bottom

In this case, we notice again that the WLSQR scheme cannot be used without a supplementary slope limiter, and the CPU time is prohibitive.

We also notice that the Hancock's time integration leads to local velocity oscillations in the air near the air-water interface. These instabilities generate negative density values. We were able to finish the computation only by disconnecting the time gradient computation when necessary. That's why we prefer the midpoint Euler time integration, which is confirmed to be more robust.

We verify that the energy model and the isothermal model are in excellent agreement. Therefore, results are presented only with the isothermal model. As demonstrated on Table 2, the saving of CPU time is important. This gain is due to several factors:

- there are less unknowns because we don't consider the energy equation;

- the Riemann solver is simpler and requires generally less Newton's iterations;

- in the energy model, the sound speed is not constant. In practice, we observe a more constraining CFL condition in the air. Thus, the time step is bigger with the isothermal model than with the energy model.

\begin{tabular}{|c|c|c|}
\hline & Isothermal model & Energy model \\
\hline first order & 0h $24 \mathrm{~min}$ & 0h $51 \mathrm{~min}$ \\
Barth & 0h $49 \mathrm{~min}$ & 2h $44 \mathrm{~min}$ \\
Euler and Barth & 1h $34 \mathrm{~min}$ & 5h 51min \\
\hline
\end{tabular}

TABLE 2. CPU time

As shown in Figure 11, where we plot the profile of the water surface, the solitary wave is well propagated. As the first order approximation is dissipative, the amplitude of the wave diminishes. But with a second order approximation in space, the 
profile is improved. We again notice that the Barth scheme gives quite good results with competitive computing time, in spite of a small distortion of the interface.

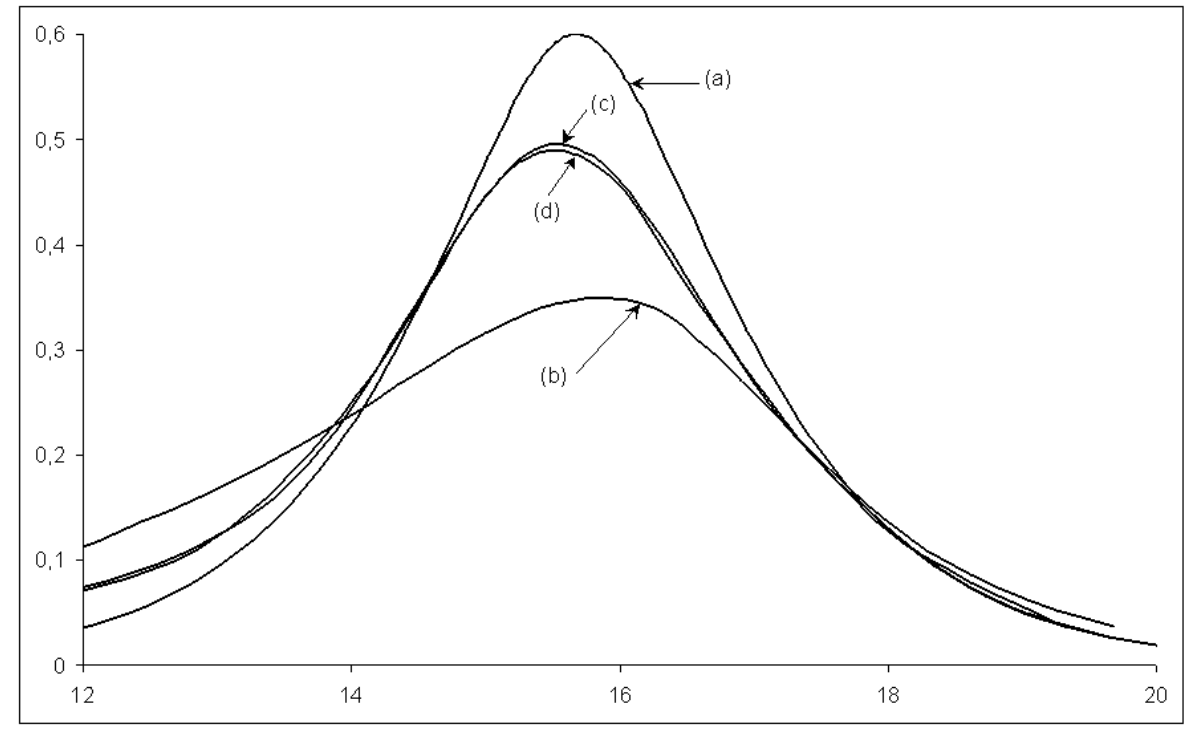

FigURE 11. Wave profile (isoline $\varphi=0.5$ ) for the isothermal model at $t=4 . s$ : (a) exact, (b) first order, (c) Barth, (d) Euler Barth

To improve the amplitude of the numerical wave, it is enough to increase the number of cells. It is demonstrated in Figure 12, where we compare the water surface computed with the isothermal model (first order in time and Barth) on a coarse grid $(600 \times 90)$ and on a finer grid $(1200 \times 180)$. 


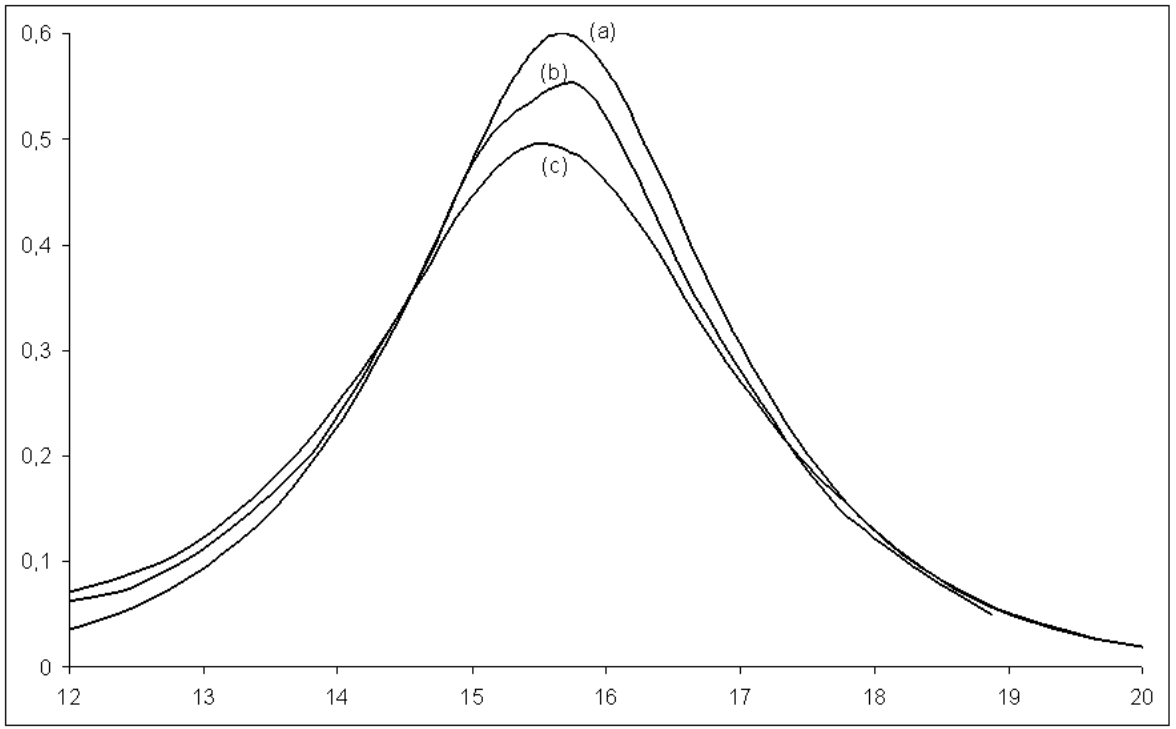

FIGURE 12. Wave profile (isoline $\varphi=0.5$ ) for the isothermal model at $t=4 . s:$ (a) exact, (b) $1200 \times 180$ cells, (c) $600 \times 90$ cells. Second order Barth limiter in space. First order in time.

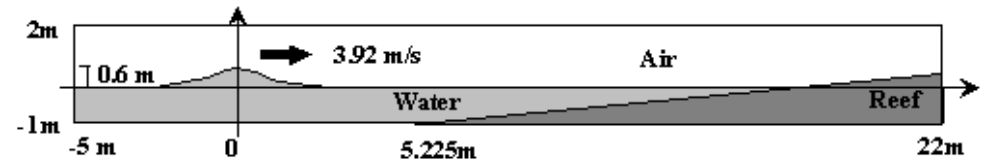

FIGURE 13. Wave breaking: solitary wave and reef

4.4. Two-dimensional wave breaking over a slope. Now, we intend to break the wave over a reef (Figure 13). The initial conditions are exactly the same as in the previous case except for the bottom. We consider at the right of $x=5.225$ a non-flat bottom equation $b(x, y)=(x-5.225) / 15$. (see 1 ), in order to break the solitary wave. The structured finite volume mesh is naturally distorted after this point.

We found a perfect agreement between the isothermal and energy model. But the isothermal model is faster (Table 3) so the investigation of 3D cases becomes more tractable.

\begin{tabular}{|c|c|c|}
\hline & Isothermal model & Energy model \\
\hline first order & 0h 39min & 1h $28 \mathrm{~min}$ \\
Barth & 1h $20 \mathrm{~min}$ & 3h $17 \mathrm{~min}$ \\
Euler and Barth & 2h 30min & 6h $14 \mathrm{~min}$ \\
\hline \multicolumn{3}{|c}{ TABLE 3. CPU time }
\end{tabular}

As for the solitary wave propagation, the first order scheme is dissipative. The breaking of the wave seems to be realistic (Figure 14). 


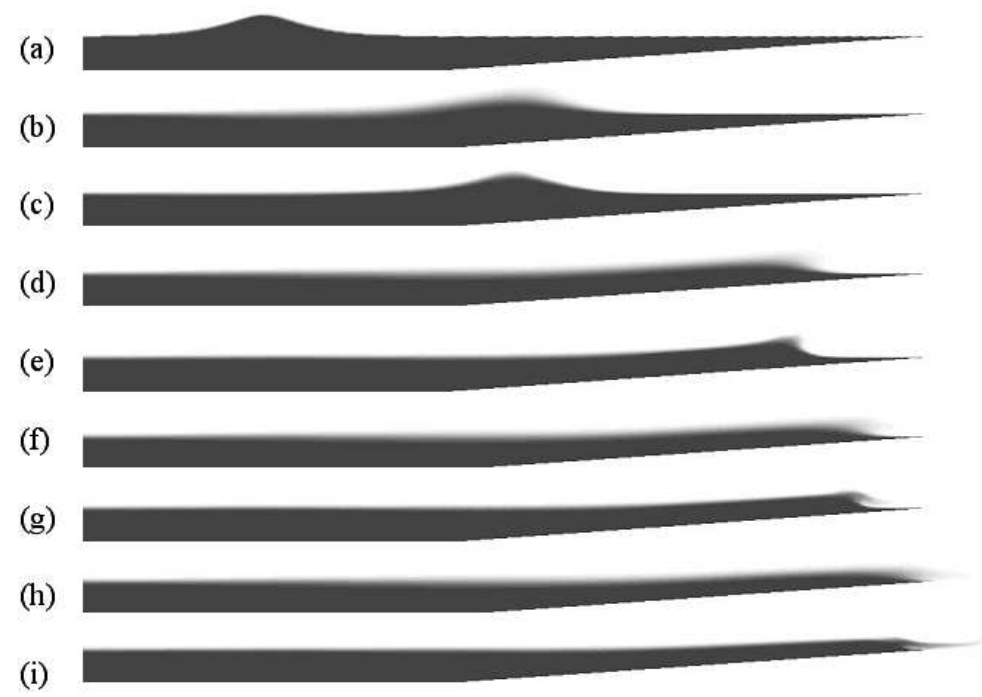

FiguRe 14. Water fraction: (a) initial data, (b) $t=2.0 \mathrm{~s}$ first order, (c) $t=2.0 \mathrm{~s}$ second order, (d) $t=4.0 \mathrm{~s}$ first order, (e) $t=4.0 \mathrm{~s}$ second order, $(\mathrm{f}) t=4.5 \mathrm{~s}$ first order, $(\mathrm{g}) t=4.5 \mathrm{~s}$ second order, (h) $t=5.0 \mathrm{~s}$ first order, (i) $t=4.0 \mathrm{~s}$ second order

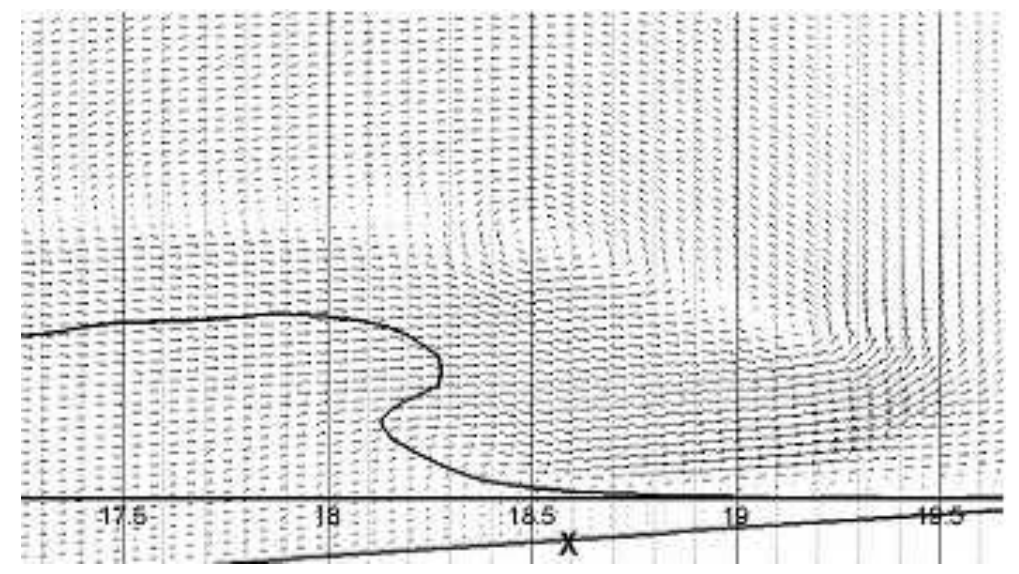

Figure 15. Isoline $\varphi=1 / 2$ and velocity vectors at time $t=4.0 \mathrm{~s}$

\section{CONCLUSION}

In this paper, we have presented a simple finite volume method to simulate twofluid incompressible flows. An artificial compressibility is introduced in order to use classical explicit schemes for compressible flows. A special tuning of the pressure law coefficients is necessary to avoid CFL restrictions and bad precision (physical preconditioning). A non-conservative treatment of the air fraction is also necessary.

We have compared several options in order to select a relevant scheme that will be now applied to 3D cases. According to our experiments we observe that: 
- the isothermal model permits to divide by 3 the CPU time compared with the energy model;

- the Barth limiter is more robust and sufficiently precise compared to the more sophisticated WLSQR limiter;

- the MUSCL-Hancock time integration is fast and precise for 1D cases. But for $2 \mathrm{D}$ computations the second order midpoint Euler time integration is more stable and thus preferable;

- the parallel version of the method is very easy to implement and lead to interesting CPU gains.

We now plan to perform 3D wave breaking computations as initiated by Biausser in [5]. We also investigate possible improvements of the precision by mesh refinement and/or higher order interpolations.

\section{REFERENCES}

[1] R. Abgrall. Generalisation of the Roe scheme for the computation of mixture of perfect gases. Recherche Aérospatiale, 6:31-43, 1988.

[2] R. Abgrall. How to prevent pressure oscillations in multicomponent flow calculations: a quasiconservative approach. Journal of Computational Physics, 125(1):150-160, 1996.

[3] T. Barberon, P. Helluy, and S. Rouy. Practical computation of axisymmetrical multifluid flows. International Journal of Finite Volumes, 1(1):1-34, 2003.

[4] C. Berthon and B. Nkonga. Behavior of the finite volumes schemes in material and numerical interfaces. In Finite Volumes for Complex Applications III (Porquerolles, 2002), pages 139146. Hermes Penton Ltd, London, 2002.

[5] B. Biausser, S. Guignard, R. Marcer, and P. Fraunié. 3D two phase flows numerical simulations by SL-VOF method. International Journal for Numerical Methods in Fluids, 45(6):581$604,2004$.

[6] G. Chanteperdrix, P. Villedieu, and Vila J.-P. A compressible model for separated twophase flows computations. In ASME Fluids Engineering Division Summer Meeting. ASME, Montreal, Canada, July 2002.

[7] J. Fürst and K. Kozel. Second and third order weighted ENO scheme on unstructured meshes. In Finite volumes for complex applications, III (Porquerolles, 2002), pages 723-730 (electronic). Lab. Anal. Topol. Probab. CNRS, Marseille, 2002.

[8] T. Gallouët, J.-M. Hérard, and N. Seguin. Some recent finite volume schemes to compute Euler equations using real gas EOS. International Journal for Numerical Methods in Fluids, 39(12):1073-1138, 2002.

[9] T. Gallouët, J.-M. Hérard, and N. Seguin. A hybrid scheme to compute contact discontinuities in one-dimensional Euler systems. M2AN. Mathematical Modelling and Numerical Analysis, 36(6):1133-1159, 2003.

[10] E. Godlewski and P.-A. Raviart. Numerical approximation of hyperbolic systems of conservation laws, volume 118 of Applied Mathematical Sciences. Springer-Verlag, New York, 1996.

[11] S. Godounov, A. Zabrodine, M. Ivanov, A. Kraı̌ko, and G. Prokopov. Résolution numérique des problèmes multidimensionnels de la dynamique des gaz. "Mir", Moscow, 1979. Translated from the Russian by Valéri Platonov.

[12] Jonathan B. Goodman and Randall J. LeVeque. On the accuracy of stable schemes for 2D scalar conservation laws. Mathematics of Computation, 45(171):15-21, 1985.

[13] J. M. Greenberg and A. Y. Leroux. A well balanced scheme for the numerical processing of source terms in hyperbolic equations. SIAM Journal on Numerical Analysis, 33(1):1-16, 1996.

[14] S. T. Grilli, J. Skourup, and I. A. Svendsen. An efficient boundary element method for nonlinear water waves. Engineering Analysis with Boundary Elements, 6(2):97-107, 1989.

[15] S. Guignard, R. Marcer, V. Rey, C. Kharif, and P. Fraunié. Solitary wave breaking on sloping beaches: 2-D two phase flow numerical simulation by SL-VOF method. European Journal of Mechanics. B. Fluids, 20(1):57-74, 2001.

[16] H. Guillard and C. Viozat. On the behaviour of upwind schemes in the low Mach number limit. Computers \& Fluids. An International Journal, 28(1):63-86, 1999. 
[17] P. Helluy, F. Golay, J.-P. Caltagirone, P. Lubin, S. Vincent, D. Drevard, R. Marcer, P. Fraunié, N. Seguin, S. Grilli, A.-C. Lesage, A. Dervieux, and O. Allain. Numerical simulations of wave breaking. M2AN. Mathematical Modelling and Numerical Analysis, 39(3):591-607, 2005.

[18] S. Karni. Multicomponent flow calculations by a consistent primitive algorithm. Journal of Computational Physics, 112(1):31-43, 1994.

[19] P.D. Lax. Hyperbolic systems of conservation laws and the mathematical theory of shock waves. In CBMS Regional Conf. Ser. In Appl. Math. 11, Philadelphia, 1972. SIAM.

[20] S. Rouy. Modélisation mathématique et numérique d'écoulements diphasiques compressibles. $\mathrm{PhD}$ thesis, Université de Toulon, December 2000.

[21] Lionel Sainsaulieu. Finite volume approximate of two-phase fluid flows based on an approximate Roe-type Riemann solver. Journal of Computational Physics, 121(1):1-28, 1995.

[22] R. Saurel and R. Abgrall. A simple method for compressible multifluid flows. SIAM Journal on Scientific Computing, 21(3):1115-1145, 1999.

[23] M. Tanaka. The stability of solitary waves. Physics of Fluids, 29:650-655, 1986.

[24] E. F. Toro. Riemann solvers and numerical methods for fluid dynamics, 2nd edition. Springer, 1999.

[25] E. Turkel. Review of preconditioning methods for fluid dynamics. Applied Numerical Mathematics, 12(1-3):257-284, 1993. Special issue to honor Professor Saul Abarbanel on his sixtieth birthday (Neveh, 1992).

[26] E. H. van Brummelen and B. Koren. A pressure-invariant conservative Godunov-type method for barotropic two-fluid flows. Journal of Computational Physics, 185:289-308, 2003.

[27] B. Van Leer. Towards the ultimate conservative difference scheme. a second order sequel to the godunov's method. Journal of Computational Physics, 32:101-136, 1979.

[28] S. Vincent, J.-P. Caltagirone, P. Lubin, and T. N. Randrianarivelo. An adaptative augmented Lagrangian method for three-dimensional multimaterial flows. Computers and Fluids, 33(10):1273-1289, 2004.

ISITV/MNC, BP 56, 83162 LA VAlette Cedex, France

E-mail address: frederic.golay@univ-tln.fr

UlP/IRMA, 7 rue Descartes, 67084 Strasbourg cedex, France

E-mail address: helluy@math.u-strasbg.fr 